\title{
Procedimiento para el Modelado de un Convertidor Boost para UPS mediante Variables de Estado
}

\author{
Procedure for Modeling a Boost Converter for UPS Using State Variables
}

\author{
Federico Gabriel D’Angiolo $^{\# 1}$, Julio Guillermo Zola ${ }^{\# 2}$

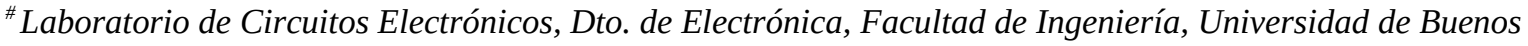 \\ Paseo Colón 850 (1063), CABA, Argentina \\ 1 fdangiolo@fi.uba.ar \\ 2 jzola@fi.uba.ar
}

Recibido: 05/02/21; Aceptado: 22/04/21

\begin{abstract}
This work describes a procedure to model a Boost converter using State Variables. An example will be taken to describe the procedure, a converter used within a UPS that must deliver a constant voltage of $400 \mathrm{~V}$ to power a bus. The input voltage of this converter is given by a set of batteries that can be fully or partially charged, so the input voltage can vary between two possible extreme values. An analysis of the Boost converter circuit will be carried out as an introduction to later carry out the corresponding modeling. This modeling will allow to analyze its stability, the variations in the output voltage in the face of changes in the input, as well as the different disturbances that may occur.
\end{abstract}

Keywords: modelling, switching converter, state variables.

Resumen-En el presente trabajo se describe un procedimiento para el modelado de un convertidor Boost utilizando variables de estado. Se tomará como ejemplo para describir el procedimiento, un convertidor utilizado en el interior de una UPS que debe entregar una tensión constante de $400 \mathrm{~V}$ para alimentar a un bus. La tensión de entrada de este convertidor viene dada por un conjunto de baterías las que pueden estar totalmente o parcialmente cargadas, razón por la cual la tensión de entrada puede variar entre dos posibles valores extremos. Se realizará como introducción un análisis del circuito convertidor Boost para luego realizar la modelización correspondiente. Esta modelización permitirá poder analizar su estabilidad, las variaciones de la tensión de salida frente a cambios en la entrada, así como las distintas perturbaciones que pudieran ocurrir.

Palabras claves: modelización, convertidor, variables de estado.

\section{INTRODUCCION}

En la actualidad muchos equipos electrónicos utilizan fuentes conmutadas para alimentar sus circuitos, sin embargo, dado que cada uno de estos equipos tienen distintos requerimientos, resulta útil analizar las distintas topologías de convertidores existentes para ver cuál es la adecuada. En particular, los sistemas de suministro de energía ininterrumpida (UPS) son capaces de alimentar cargas que necesitan una constante alimentación, es decir, una alimentación que no se vea perturbada por ningún motivo. Algunos ejemplos de este tipo de carga pueden ser equipos médicos, datacenters, equipos de procesamiento industrial y equipos de gestión en línea los cuales, por ejemplo, ante un corte de la alimentación general de $220 \mathrm{~V}$, deben seguir funcionando.

Por esta razón, las UPS generalmente se encuentran equipadas con un conjunto de baterías que son las encargadas de continuar con el suministro de energía ante un corte en la alimentación general.

En el interior de una UPS se encuentra un circuito que toma la tensión generada por estas baterías y la eleva a otra de $400 \mathrm{~V}$ para alimentar la electrónica interna que será capaz de seguir con el proceso de suministro de energía a la carga. Este circuito elevador, resulta ser una convertidor Boost.

En particular, este tipo de convertidor permite elevar la tensión a su salida con respecto a la de su entrada - Fig.1-. Esta elevación de tensión implica una disminución de la corriente de salida con respecto a la de entrada, ya que la potencia puesta en juego debe mantenerse idealmente constante [1].

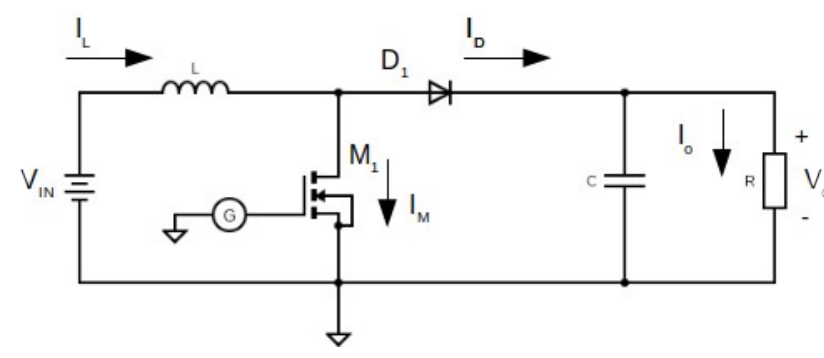

Fig. 1. Convertidor Boost.

Los componentes que almacenan energía (inductor y capacitor), serán los encargados junto con los dispositivos semiconductores, de elevar la tensión de entrada a 400V.

El transistor $\mathrm{M}_{1} \mathrm{y}$ el diodo $\mathrm{D}_{1}$ actúan como dispositivos conmutadores en momentos distintos, es decir, mientras uno 
de ellos se encuentra en modo de corte (OFF), el otro se encuentra en modo de conducción (ON), es decir, con una caída de tensión de 0V. Por esta razón, para comprender el funcionamiento del circuito de manera sencilla, se reemplazará a cada uno por una llave ideal. Dado que cada uno de estos dispositivos conmutan en momentos distintos, se necesita de una fuente o generador que los pueda sincronizar. En este caso, quien realiza esta acción es el bloque $\mathbf{G}$, el cual genera una señal modulada por ancho de pulso (pulse width modulation, PWM). La frecuencia $\mathrm{y}$ ancho del pulso dependen de las especificaciones y de su posterior cálculo. Como se puede ver en la Fig.1, en el caso del diodo, el mismo actuará en directa (conducción) o en inversa (corte), de acuerdo a la diferencia de potencial que exista entre sus terminales en cada momento.

La tensión de entrada $\mathrm{V}_{\mathrm{IN}}$, (se la denominará Vi en las fórmulas para simplificar los subíndices) será elevada por medio del convertidor a la tensión de salida $\mathrm{V}_{\mathrm{O}}$, la cual para nuestro ejemplo de análisis es de 400V.

Para el presente caso, $\mathrm{V}_{\text {IN }}$ será suministrada por un conjunto baterías y tendrá un rango comprendido entre $100 \mathrm{~V}$ y $219 \mathrm{~V}$, según sea el estado de carga de dichas baterías, es decir, si están totalmente cargadas la tensión de entrada será de $219 \mathrm{~V}$ mientras que si se encuentran con carga mínima, la tensión de entrada será de $100 \mathrm{~V}$. Por otra parte, dado que la potencia requerida es de $5 \mathrm{KW}$, la corriente de salida será de 12,5A.

Admitiremos para nuestro modelo que el convertidor bajo análisis trabaja en Modo Continuo, con lo cual la relación entre la tensión de entrada y la de salida, viene dada por:

$$
V_{0}=\frac{V_{i}}{1-D}
$$

Siendo:

$\mathrm{D}=$ Ciclo de trabajo.

$\mathrm{V}_{\mathrm{i}}=$ Tensión de entrada del convertidor.

$\mathrm{V}_{\mathrm{O}}=$ Tensión de salida del convertidor.

\section{MODO DE FUNCIONAMIENTO}

El Convertidor tiene dos modos de funcionamiento: Modo Continuo (CM) y Modo Discontinuo (DM). El primer modo presenta la ventaja de que la tensión de salida no depende de la carga mientras que, en el DM la tensión de salida sí depende de la carga. A su vez, en el funcionamiento del DM, la corriente que atraviesa el inductor se vuelve nula antes de que comience el próximo ciclo de conmutación, lo cual es un beneficio desde el punto de vista de la disipación pero, cuando se implementa el circuito, resulta muy importante que no existan capacidades parásitas importantes dado que de ser así se puede generar una oscilación amortiguada al producirse la conmutación ("ringing”) el cual es perjudicial para las Interferencias Electromagnéticas (EMI). La descripción que sigue a continuación, estará basada en el primer modo.

El funcionamiento del circuito de la Fig.1, se puede comenzar describiendo para el momento en el que el transistor $\mathrm{M}_{1}$ se encuentra en conducción (debido a que el generador $\mathrm{G}$ elevó suficientemente la tensión del terminal de Gate) y el diodo $\mathrm{D}_{1}$ se encuentra en inversa. A este momento lo podemos denominar $\mathrm{t}_{\mathrm{ON}}$. En base a esto, se observa que circula corriente por el inductor, la cual es provista por la fuente de entrada, $\mathrm{V}_{\mathrm{IN}}$. Dado que la relación entre corriente y tensión en un inductor viene dada por la expresión:

$$
v_{L}(t)=\mathrm{L} \cdot \frac{d i_{L}(t)}{d t}
$$

De la ecuación anterior se puede ver que, para obtener la evolución de la corriente, se puede integrar, teniendo en cuenta el valor de la corriente inicial. Luego, dado que en este caso, $\mathrm{V}_{\mathrm{L}}=\mathrm{V}_{\mathrm{IN}}$, se puede obtener:

$$
i_{L}(t)=\frac{1}{L} \cdot \int V_{I .} d t+i_{L}(0)
$$

Dado que $\mathrm{V}_{\mathrm{i}}$ es constante, se llega entonces a que:

$$
i_{L}(t)=\frac{1}{L} \cdot V_{I} \cdot t+i_{L}(0)
$$

De la expresión anterior, se puede ver que la corriente en el inductor aumenta linealmente con el tiempo hasta que $\mathrm{M}_{1}$ deje de estar en conducción debido a que el generador $\mathrm{G}$ cambia de valor, es decir, el transistor pasa a estado de corte. Se puede decir que en este proceso, el inductor almacena la energía en forma de campo magnético.

Luego, el siguiente estado del circuito se da cuando $\mathrm{M}_{1}$ entra en corte y el diodo $\mathrm{D}_{1}$ comienza a conducir (se polariza en directa). En este intervalo de tiempo, el inductor descarga la energía almacenada sobre el capacitor y la carga. Despreciando la caída de tensión en el diodo $\mathrm{D}_{1}$ la corriente en el inductor queda de la siguiente forma:

$$
i_{L}(t)=\frac{1}{L} \cdot\left(V_{I}-V_{O}\right) \cdot t+i_{L}(0)
$$

Observando la expresión anterior, se puede ver que la corriente en el inductor decae linealmente dado que $\mathrm{V}_{\text {o }}$ es mayor que $\mathrm{V}_{\mathrm{IN}}$, tal como se observa del principio de funcionamiento de este tipo de convertidores -ver ecuación (1) -

\section{MODELIZACIÓN}

\section{A. Análisis del Circuito Convertidor}

El convertidor analizado se lo puede estudiar como un sistema en lazo abierto, siendo la señal de entrada la tensión $\mathrm{V}_{\mathrm{i}}\left(\mathrm{o} \mathrm{V}_{\mathrm{IN}}\right)$ y su salida la tensión $\mathrm{V}_{\mathrm{o}}$. En este caso, no hay que olvidar que el valor de $\mathbf{D}$, se mantiene constante para que la tensión de salida no se vea modificada (ante un único valor de $\mathrm{Vi}$ ). Es decir, $\mathrm{V}_{\mathrm{i}}$ no es la única señal de entrada al convertidor sino que también se encuentra otra que viene dada por la señal proveniente del modulador por ancho de pulso (G) -Fig. 1-. Por esta razón el lazo abierto será modelado con una señal de entrada que será d y una señal de salida que será $\mathbf{V}_{\mathbf{0}}$. -Fig. 2-. 


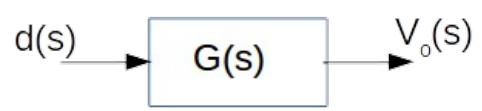

Fig. 2. Convertidor Boost.

Siendo:

d(s): Señal de entrada proveniente del PWM, en el dominio de Laplace.

$\mathrm{V}_{\mathrm{o}}(\mathrm{s})$ : Tensión de salida del convertidor, en el dominio de Laplace.

Es importante aclarar que, al cerrar el lazo, se elimina este generador de señal $\mathbf{G}$ ya que precisamente este lazo tiene como objetivo muestrear tensión a la salida e inyectar al gate del transistor una señal del tipo PWM para que, modulando el ciclo de trabajo, se pueda mantener a $\mathrm{V}_{\mathrm{O}}$ con la menor variación posible.

Una vez modelizado el convertidor, se podrá cerrar el lazo para poder estabilizar $\mathrm{V}_{\mathrm{O}}$ frente a perturbaciones que puedan existir. Para esto, se opta por usar un lazo de muestreo y suma de tensión [2] - Fig. 3 -.

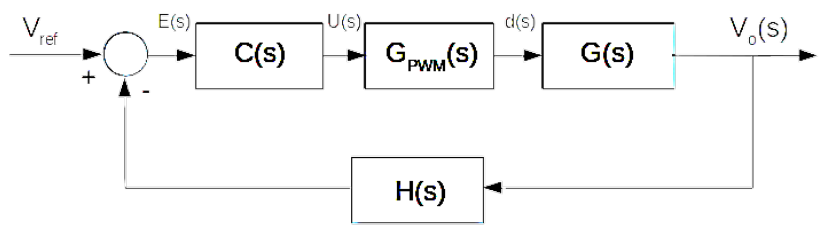

Fig. 3. Modelización del Convertidor en lazo cerrado.

Los bloques con Función de Transferencia son:

C(s): Función transferencia del controlador (puede ser del tipo Proporcional)

GPWM(s): Función Transferencia del modulador PWM

G(s): Función Transferencia del convertidor

H(s): Función Transferencia del realimentador

Las señales descritas son:

E(s): Señal de error, surge como la diferencia entre la tensión de referencia y la que proviene del realimentador.

U(s): Señal proveniente del controlador.

d(s): Señal proveniente del modulador PWM.

Se procederá a modelizar cada una de estas Funciones, empezando por $\mathrm{G}(\mathrm{s})$, ya que es la que se desea analizar tanto en lazo abierto como en lazo cerrado. Luego, se procederá a modelizar los bloques restantes [3].

\section{B. Modelización del Convertidor en Lazo Abierto}

Se utilizará la técnica de "Modelo con Variable Promedio" [9]. Es decir, dado que el funcionamiento del Convertidor no es lineal se acude a un método que pueda promediar los dos estados bajo los que funciona el convertidor. Para lograr el modelo deseado, se hace una breve descripción de los modos de funcionamiento de dicho convertidor a lo largo de un período de la señal y se toman sus circuitos equivalentes.

Se tomará como circuito bajo estudio, el de la Fig. 4:

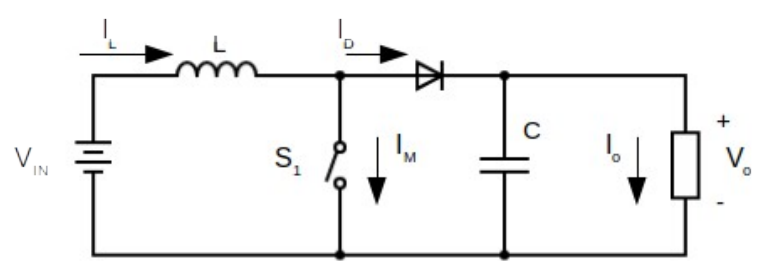

Fig. 4. Convertidor Boost con modelos ideales de capacitores e inductores.

Es conveniente aclarar que la modelización se puede realizar tanto con los modelos ideales o reales de sus componentes (por ejemplo teniendo en cuenta la resistencia, capacidad e inductancia parásita de los componentes reactivos). Para el caso del presente trabajo, se prefiere considerar los modelos ideales para comprender la metodología en el análisis. Sin embargo, se pueden usar modelos más complejos de manera que el estudio se asemeje lo más posible al comportamiento real del convertidor [4-8]. Cabe aclarar que el alcance de este trabajo es realizar un análisis básico que permita comprender el uso de las variables de estado para modelizar un convertidor Boost y no tiene en cuenta otros aspectos del circuito que complejizarían el modelo, tales como los componentes parásitos del arreglo circuital. El análisis de la influencia de estos componentes, que ampliaría las ecuaciones y por lo tanto el modelo a construir, resultará ser la base de un futuro trabajo donde además pueda hacerse el estudio de estabilidad de dicho convertidor.

Para este análisis, se admiten ideales a los elementos de conmutación. Es decir, el transistor de conmutación $\mathrm{M}_{1}$ se lo sustituye por una llave ideal $\mathrm{S}_{1}$. Asimismo, el diodo $\mathrm{D}_{1}$ actúa como ideal.

El intervalo ton durante el cual, el inductor almacena energía, se lo denominará MODO ON. Luego, el intervalo $t_{\mathrm{OFF}}$, cuando el inductor libera la energía almacenada, será el MODO OFF. En cada uno de estos modos el convertidor tendrá su equivalente circuital y por ende una representación matemática, descripta a través de variables de estados.

Se parte de las ecuaciones de estado del circuito, en cualquiera de sus modos, considerando un vector de estado compuesto por las variables corriente en la inductancia $\left(\mathrm{i}_{\mathrm{L}}\right) \mathrm{y}$ tensión en el capacitor de salida $\left(\mathrm{v}_{\mathrm{c}}=\mathrm{v}_{\mathrm{o}}\right)$, (6). 


$$
x=\left(i_{L} v_{o}\right)^{T}
$$

Se toman estas variables dado que en el circuito de la Fig. 1, se encuentran dos elementos que almacenan energía: el capacitor, que almacena energía en forma de campo eléctrico y el inductor que almacena energía en forma de campo magnético.

En base a esto, a continuación se analizan los modelos matemáticos para cada intervalo, según la modelización descripta en [9].

\section{Primer modo: Modo ON}

Este análisis comienza teniendo en cuenta que la llave $S_{1}$ (transistor) se encuentra cerrada y el diodo $\mathrm{D}_{1}$, en inversa. El circuito equivalente es el que se muestra en la Fig. 5

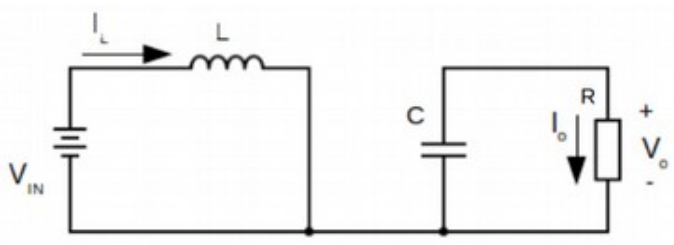

Fig. 5. Circuito equivalente del convertidor en Modo ON.

Planteando las ecuaciones de Kirchoff de tensiones y corrientes en las mallas que contienen a L y a C (KVL y KVC, respectivamente) sobre el circuito de la Fig. 5 y utilizando como variables de estado a la corriente en el inductor $\left(\mathrm{i}_{\mathrm{L}}\right)$ y a la tensión en el capacitor $\left(\mathrm{v}_{\mathrm{C}}\right)$, se tiene que:

$$
\dot{x}=A_{1} \cdot x+B_{1} \cdot v_{i}
$$

$$
\begin{aligned}
& {\left[\begin{array}{l}
\frac{d_{i L}}{d t} \\
\frac{d_{v c}}{d t}
\end{array}\right]=\left[\begin{array}{cc}
0 & 0 \\
0 & \frac{-1}{C \cdot R}
\end{array}\right] \cdot\left[\begin{array}{c}
i_{L} \\
v_{c}
\end{array}\right]+\left[\begin{array}{c}
\frac{1}{L} \\
0
\end{array}\right] \cdot v_{i}} \\
& v_{o}=C_{1} \cdot x \\
& v_{o}=\left[\begin{array}{ll}
0 & 1
\end{array}\right] \cdot\left[\begin{array}{c}
i_{L} \\
v_{c}
\end{array}\right]
\end{aligned}
$$

\section{Segundo modo: Modo OFF}

El análisis en este modo, se inicia con la llave $S_{1}$ (transistor) abierta y el diodo $\mathrm{D}_{1}$, en directa. De esta forma, el circuito equivalente es el indicado en la Fig. 6

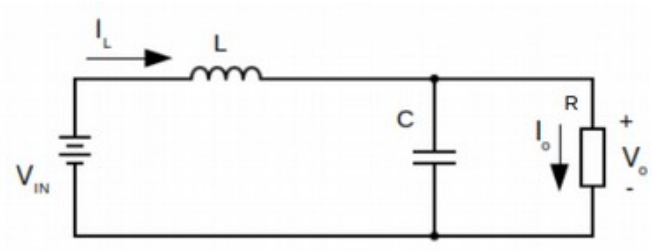

Fig. 6. Circuito equivalente del Convertidor en Modo OFF.

Planteando KVL y KVC, se tiene que:

$$
\dot{x}=A_{2} \cdot x+B_{2} \cdot v_{i}
$$

$$
\begin{array}{r}
{\left[\begin{array}{c}
\frac{d_{i L}}{d t} \\
\frac{d_{v c}}{d t}
\end{array}\right]=\left[\begin{array}{cc}
0 & \frac{-1}{L} \\
\frac{1}{C} & \frac{-1}{C \cdot R}
\end{array}\right] \cdot\left[\begin{array}{l}
i_{L} \\
v_{c}
\end{array}\right]+\left[\begin{array}{c}
\frac{1}{L} \\
0
\end{array}\right] \cdot v_{i}} \\
v_{o}=C_{2} \cdot x \\
v_{o}=\left[\begin{array}{ll}
0 & 1
\end{array}\right] \cdot\left[\begin{array}{c}
i_{L} \\
v_{C}
\end{array}\right]
\end{array}
$$

Teniendo formadas las matrices para el convertidor en cada uno de sus estados, se procede a promediarlas y linealizarlas, quedando:

$$
\begin{gathered}
{\left[\begin{array}{l}
\frac{\tilde{d}_{i L}}{d t} \\
\frac{\widetilde{d}_{v c}}{d t}
\end{array}\right]=\left[\begin{array}{cc}
0 & -\frac{1-D}{L} \\
\frac{1-D}{C} & -\frac{1}{C \cdot R}
\end{array}\right] \cdot\left[\begin{array}{l}
\widetilde{i}_{L} \\
\widetilde{v}_{C}
\end{array}\right]+\left[\begin{array}{ll}
\frac{1}{L} & \frac{V_{i}}{L \cdot(1-D)} \\
0 & -\frac{V_{i}}{R \cdot C \cdot(1-D)^{2}}
\end{array}\right] \cdot\left[\begin{array}{c}
\widetilde{v} i \\
\widetilde{d}
\end{array}\right]} \\
v_{o}=\left[\begin{array}{ll}
0 & 1
\end{array}\right] \cdot\left[\begin{array}{l}
i_{L} \\
v_{C}
\end{array}\right]
\end{gathered}
$$

Con esta modelización matemática, el diagrama en bloques de la fuente queda entonces como se muestra en la Fig. 7.

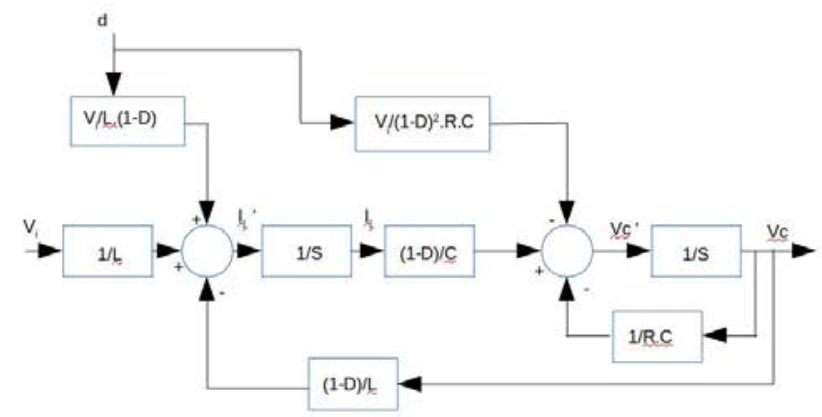

Fig. 7. Diagrama en bloques de la fuente conmutada. Vc es la tensión sobre el capacitor, que coincide con la tensión de salida. 


\section{Modelización del Convertidor en Lazo cerrado}

Retomando la Fig. 3, se puede observar que en lazo cerrado, se encuentran tres bloques más, $\mathrm{G}_{\mathrm{PwM}}(\mathrm{s}), \mathrm{H}(\mathrm{s})$ y $\mathrm{C}(\mathrm{s})$. Se analizará primeramente $\mathrm{G}_{\mathrm{PWM}}(\mathrm{s})$.

\section{Modelización del modulador PWM}

El bloque modulador de PWM, tiene como objetivo generar una señal cuadrada pero con un ciclo de trabajo variable. Para lograr esto último, el modulador toma la señal proveniente del controlador C(s) y la compara contra una señal diente de sierra de amplitud y frecuencia fija. En base a esto, la modelización de este bloque se puede obtener como: [9]

$$
G_{P W M}=\frac{1}{V_{M}}
$$

Siendo:

$\mathrm{G}_{\mathrm{PWM}}(\mathrm{s})$ = Función Transferencia del bloque modulador.

$\mathrm{V}_{\mathrm{M}}=$ tensión pico de la señal diente de sierra.

La tensión $V_{M}$ se puede tomar de circuitos integrados de fuentes conmutadas comerciales. Como se puede ver, $\mathrm{G}_{\mathrm{PWM}}(\mathrm{s})$ no depende de la frecuencia.

\section{E. Modelización del bloque realimentador}

El bloque realimentador muestrea a $\mathrm{V}_{\text {o }} \mathrm{y}$ suma tensión a la entrada del sistema realimentado, ya que se debe comparar con la tensión de referencia $\mathrm{V}_{\mathrm{REF}}$. En base a esto, el bloque $\mathrm{H}(\mathrm{s})$ se puede escribir como:

$$
H(s)=\frac{V_{f}}{V_{o}}
$$

Siendo:

H(s) = Función Transferencia del bloque realimentador.

$\mathrm{V}_{\mathrm{f}}=$ tensión de salida de $\mathrm{H}(\mathrm{s})$.

$\mathrm{V}_{\mathrm{o}}=$ tensión de salida del convertidor.

En este caso, el cálculo de $\mathrm{H}(\mathrm{s})$ será realizado para régimen permanente. Es decir, $\mathrm{V}_{\mathrm{o}}=400 \mathrm{~V}$ y $\mathrm{V}_{\mathrm{f}}$ cercana a la tensión de referencia. Esta última se la puede tomar de los circuitos integrados utilizados en fuentes conmutadas comerciales. Con esto se tiene que:

$$
\mathrm{V}_{\mathrm{o}}=400 \mathrm{~V} ; \mathrm{V}_{\mathrm{REF}}=2,5 \mathrm{~V}
$$

El valor de $\mathrm{V}_{\mathrm{REF}}$ se tomó del circuito integrado TL494, aunque muchos dispositivos toman una tensión similar.

\section{F. Modelización del bloque Controlador}

Este bloque tiene como función, generar una señal apta para que el Convertidor pueda mantener la tensión de salida en 400V. Para lograr esto, toma la señal de error proveniente de la diferencia entre la señal de referencia $\left(\mathrm{V}_{\mathrm{REF}}\right)$ y la que entrega el bloque realimentador $\mathrm{H}(\mathrm{s})$ (Ver Fig.3). Es decir que la transferencia de este bloque será:

$$
C(s)=\frac{U(s)}{E(s)}
$$

Siendo:

C(s) = Función Transferencia del Controlador.

$\mathrm{E}(\mathrm{s})=$ Señal de Error, en el dominio de Laplace.

$\mathrm{U}(\mathrm{s})=$ Señal de Control en el dominio de Laplace.

El Controlador a utilizar es uno del tipo Proporcional, dada las prestaciones que ofrece para Convertidores. Dicho controlador se puede representar matemáticamente de la siguiente forma:

$$
C(s)=K_{p}
$$

Siendo $\mathrm{K}_{\mathrm{P}}$ la Constante Proporcional.

Dado que el objeto de estudio de este artículo no resulta ser la sintonización del Controlador, se opta por utilizar el método Ziegler - Nichols. Esto es, para obtener mejores prestaciones, resulta conveniente realizar el estudio de dicha sintonización y la posible utilización de una parte integradora y otra derivativa, dentro del mismo Controlador.

\section{RESULTADOS}

Se procede a mostrar en la Fig. 8 el esquema final:

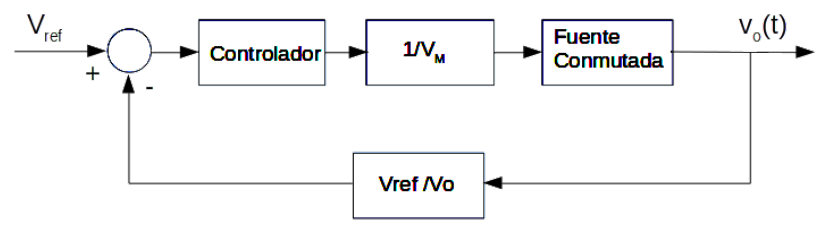

Fig. 8. Diagrama en bloques del modelo Final.

A los efectos demostrativos del diagrama de la Fig.8, se propone una simulación hecha en Scilab con algunos valores para el Convertidor, de manera de poder simular la relación entre entrada y salida del circuito.

Valores propuestos: $\mathrm{L}=760 \mu \mathrm{H} ; \mathrm{C}=47 \mu \mathrm{F}$; $\mathrm{R}=32 \Omega \mathrm{H}=6,25 \cdot 10^{-3} \mathrm{~V} / \mathrm{V} ; \mathrm{K}_{\mathrm{P}}=0,1$

La imagen que se muestra a continuación, resulta ser la salida del convertidor ante una excitación del tipo escalón en $\mathrm{V}_{\mathrm{REF}}$ de $2,5 \mathrm{~V}$. 


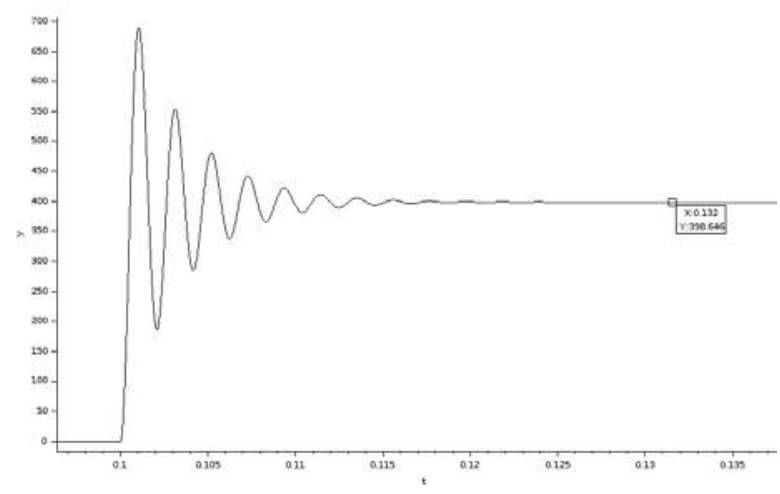

Fig. 9. Tensión de salida vs tiempo.

En la Fig. 9 se puede ver que con el valor de $K_{P}$ elegido, la tensión de salida se mantiene aproximadamente en 398,6V (en estado estable), pero en contraposición se tiene un transitorio con oscilaciones cuyas amplitudes alcanzan los $700 \mathrm{~V}$, lo cual no resulta beneficioso para el circuito donde se va a utilizar este convertidor. De acuerdo a las exigencias del circuito, se puede agregar una parte Integral al controlador para disminuir el error en estado estable y llegar a los 400V, teniendo en cuenta los efectos que puede agregar la parte integral [10]. Por otro lado, para disminuir el sobrepico, se puede agregar un lazo de corriente interno al sistema [11].

\section{CONCLUSIONES}

El estudio presentado no pierde de vista los bloques que componen el lazo cerrado sobre el cual se encuentra la fuente lo cual permite obtener un modelo completo del comportamiento de la fuente en lazo cerrado para evaluar en futuros estudios, como por ejemplo, el comportamiento dinámico y los posibles controladores que lo puedan integrar.

Teniendo en cuenta la respuesta en la tensión de salida luego de ajustar el Controlador Proporcional, se observa la necesidad de agregar otro controlador para el sensado de la corriente en el inductor. Al tener modelizadada a esta variable, se puede proponer como trabajo a futuro la sintonización de un controlador para lograr un menor sobrepico en la tensión de salida.

\section{AGRADECIMIENTOS}

Este trabajo fue llevado a cabo desde el Laboratorio de Circuitos Electrónicos de la Facultad de Ingeniería de la Universidad de Buenos Aires en conjunto con la Empresa Crexel S.R.L.

\section{REFERENCIAS}

[1] N. Moham, T. M. Undeland, y W. Robins, "Power Electronics, Converters, Applications, and Design,” John Wiley \& Sons, 2003.

[2] K. Ogata, Ingeniería de Control Moderna, 5ta ed., 2010. ISBN: 97884-8322-660-5.

[3] CH. Huang, Y.Chuang and Y. Ke, "Design of Closed-loop Buckboost Converter for LED Driver Circuit” Department of Electrical Engineering. National Penghu University of Science and Technology. Penghu, Taiwan, R.O.C.

[4] V. Sood, H.Abdel-Gawad, "Small-Signal Analysis of Boost Converter, including Parasitics, operating in CCM"
[5] X. Cheng, G. Xie, "Full Order Models and Simulation of Boost Converters Operating in DCM". International Conference on Electronic Computer Technology. 2009

[6] D.Ng, W. Wong, K.Wan and D.Kwong “An Efficient TransferFunction-Based Approach for the Fast Tunning and Design of DCDC Converters”.2nd IEEE Conference on Industrial Electronics and Applications. 2007

[7] L.Guo, "Implementation of Digital PID Controllers for DC-DC Converters using Digital Signal Processors" IIEEE EIT 2007 Proceedings

[8] V. Sood, H.Abdel-Gawad, "Small-Signal Analysis of Boost Converter, including Parasitics, operating in CCM

[9] J.C. Floriani, Fuentes Conmutadas. Análisis y Diseño, 2010: ISBN: 987-9406-45-1.

[10] Texas Instruments, "Practical Feedback Loop Analysis for VoltageMode Boost Converter”. Application Report SLVA633- January 2014.

[11] A. Bersani, "Switch Mode Power Supply (SMPS) Topologies (Part II)”. AN1207.Microchip Technology Inc.. 